\title{
A STUDY OF THE SYMMETRY OF FIELD PATTERNS OF ELLIPTIC HORNS FED BY ELLIPTIC WAVEGUIDES
}

\author{
Smain Amari* and Jens Bornemann \\ Laboratory for Lightwave Electronics, Microwaves and Communications \\ (LLiMiC) \\ University of Victoria, Victoria, B.C., Canada V8W 3P6
}

\section{INTRODUCTION}

Horn antennas are widely used as feeding sytems in a variety of antennas in space communications and astronomy. Conical and pyramidal horn antennas, although lending themselves to reasonably simple design procedures, yield asymmetric field patterns. Corrugations along the inner surface of the horn are introduced to reduce this asymmetry as discussed in [1]. While an elliptic horn fed by an elliptic waveguide is also known to exhibit symmetric field patterns, its theoretical treatment was always perceived as too time-consuming.

With the availability of faster and larger digital computers, however, the computation of Mathieu functions, in terms of which the eigenmodes of elliptic waveguides can be expressed, is a practical undertaking. When the elliptic horn is operated in the frequency range, where only its dominant mode, $\mathrm{TE}_{\mathrm{c} 11}$, is propagating, only a single set of expansion coefficients are in fact needed, as both Mathieu $\left(c_{1}\right)$ and modified Mathieu ( $\left.\mathrm{Ce}_{1}\right)$ functions share the same set of expansion coefficients [2]. These expansion coefficients can be calculated once at the beginning of the computations, they are tead from the computer memory every time values of Mathien functions are needed. Taking advantage of this property considerably speeds up the calculations. In addition, exceedingly accurate expressions for the cutoff frequencies of elliptic waveguides versus eccentricity were recently published in [3].

The analysis presented here follows the modified analysis of conical horns presented in [4]. The computation of the phase difference at the aperture is, however, more complicated, as one cannot define a single apex for all wavefronts as in the case of a conical horn, except when the eccentricity of the horn is kept constant and equal to that of the feeding waveguide. In the following section, we show how the phase difference at the elliptic aperture is computed.

\section{THEORY}

The calculation of the phase aperture for the elliptic horn is similar to that for the conical hom [4]. Therefore, only the salicnt differences are highlighted here.

The geometry of the horn is shown in Fig. 1. The horn is of length $H$, the semi-major axes of the feeding guide and the aperture are $a_{0}$ and $a_{1}$, respectivcly, with eccentricities $e_{0}$ and $e_{1}$. We assume that the eccentricity of the hom and its major axis vary linearly with $z$. The phase shift between the "phase center" and the aperture is the sum of two terms 


$$
\Phi_{c}(\eta)=\frac{2 \pi}{\lambda_{\mathrm{gTE}_{\mathrm{c} 11}}} h_{0}(\eta)+\int_{0}^{\mathrm{H}} \beta_{\mathrm{TE}_{\mathrm{c} 11}} \mathrm{dz}
$$

Here, $\lambda_{\mathrm{gTE}}$ is the wavelength of the fundamental $\mathrm{TE}_{\mathrm{cl1}}$ mode in the feeding waveguide, and $\beta$ is the propagation constant along the $z$-axis in the horn. The quantity $h_{0}(\eta)$ determines the location of the apex and depends on the position along the circumference of the aperture. After some manipulation, we get

$$
\left(\frac{h_{0}}{H}\right)^{2}=\frac{a_{0}^{2}\left(1-e_{0}^{2}\right) \frac{1+\left(1-e_{1}^{2}\right) T^{2}}{\left(1-e_{0}^{2}\right)+\left(1-e_{1}^{2}\right) T^{2}}}{a_{1}^{2}\left(1-e_{1}^{2}\right) S^{2}-a_{0}^{2}\left(1-e_{0}^{2}\right) \frac{1+\left(1-e_{1}^{2}\right) T^{2}}{\left(1-e_{0}^{2}\right)+\left(1-e_{1}^{2}\right) T^{2}}}
$$

where $T=\tan (\eta)$ and $S=\sin (\eta)$.

The case of a conical horn corresponds to $\mathrm{e}_{0}=\mathrm{e}_{1}=0$, and $\mathrm{h}_{0}=\mathrm{a}_{0} \mathrm{H} /\left(\mathrm{a}_{1}\right.$ - $\left.\mathrm{a}_{0}\right)$. Letting $e_{0}=e_{1}=0$ in (2) yields this result. Also, if the eccentricity does not depend on 7, the same result holds and is obtained from (2) by letting $e_{0}=e_{1}$.

The second term in equation (1) can be rewritten as

$$
\Phi_{\text {horn }}=\frac{2 \pi H}{\lambda} \int_{0}^{1} \sqrt{1-\left[\frac{\lambda}{\mathrm{a}(\mathrm{z} / \mathrm{H}) \mathrm{F}(\mathrm{e}(\mathrm{z} / \mathrm{H}))}\right]^{2}} \mathrm{dz}
$$

where the function $\mathrm{F}[\mathrm{e}(\mathrm{z})]=\stackrel{0}{\lambda_{\mathrm{c}}} \mathrm{c}(\mathrm{z}) / \mathrm{a}(\mathrm{z})$ is given in. [3]. This integral is evaluated numerically using standard quadratures for example. The phase shift at any point of the aperture, in terms of its elliptic coordinates $(\xi, \eta)$, can be written as

$$
\Phi(\xi, \eta)=\Phi_{c}\left(\frac{e_{1} a_{1}}{H+h_{0}(\eta)}\right)^{2}-\frac{(\cosh \xi)^{2}-(\sin \eta)^{2}}{1+\sqrt{1+\left(\frac{e_{1} a_{1}}{H+h_{0}(\eta)}\right)^{2}\left[(\cosh \xi)^{2}-(\sin \eta)^{2}\right]}}
$$

The far-field components of the clectric field are given by expressions, which are similar to those of the conical horn, namely

$$
\begin{aligned}
& \mathrm{E}_{\theta} \propto\left(1+\frac{\beta_{\mathrm{TE}} \mathrm{cl1}}{\mathrm{k}} \cos \theta\right)\left(\mathrm{I}_{1} \sin \phi-\mathrm{I}_{2} \cos \phi\right) \\
& \mathrm{E}_{\phi} \propto\left(\cos \theta+\frac{\beta_{\mathrm{TE}}}{\mathrm{k}}\right)\left(\mathrm{I}_{1} \cos \phi+\mathrm{I}_{2} \sin \phi\right)
\end{aligned}
$$

Integrals $\mathrm{I}_{1}$ and $\mathrm{I}_{2}$ are given by

$\xi_{0} 2 \pi$

$$
\begin{gathered}
I_{1}=\int_{0} \int_{0}\left[\mathrm{Ce}_{1}(\xi) \mathrm{ce}_{1}^{\prime}(\eta) \sinh \xi \cos \eta+\mathrm{Ce}_{1}{ }_{1}(\xi) \mathrm{ce}_{1}(\eta) \cosh \xi \sin \eta\right] \\
\cdot \mathrm{e}^{\mathrm{j}\left\{\mathrm{ka}_{1} \mathrm{e}_{1} \sin \theta[\cosh \xi \cos \eta \cos \phi+\sinh \xi \sin \eta \sin \phi]-\Phi(\xi, \eta)\right\}} \mathrm{d} \eta \mathrm{d} \xi
\end{gathered}
$$




$$
\begin{aligned}
& \xi_{0} 2 \pi \\
& I_{2}=\iint\left[\mathrm{Ce}_{1}(\xi) \operatorname{ce}_{1}^{\prime}(\eta) \cosh \xi \sin \eta-\mathrm{Ce}_{1}^{\prime}(\xi) \operatorname{ce}{ }_{1}(\eta) \sinh \xi \cos \eta\right] \\
& 00 \cdot \mathrm{e}^{j\left\{k a_{1} e_{1} \sin \theta[\cosh \xi \cos \eta \cos \phi+\sinh \xi \sin \eta \sin \phi]-\Phi(\xi, \eta)\right\}} \mathrm{d} \eta \mathrm{d} \xi
\end{aligned}
$$

$\xi_{0}$ is related to the eccentricity of the aperture $e_{1}$ by $\cosh \xi_{0}=1 / e_{1}$.

The Mathieu functions $\mathrm{Ce}_{1}$ and $\mathrm{ce}_{1}$ as well as their derivatives are computed from their expansion coefficients in terms of Bessel functions, or simply their Fourier series, since the two are essentially equal [2]. These coefficients are calculated only once and stored. The integrals in equations (7), (8), are evaluated numerically using Gauss-Legendre quadratures.

\section{RESULTS}

The field pattern of an elliptic horn antenna fed by an elliptic waveguide were determined using the present analysis. The dimensions of the antenna, which is operated at $1.21 \mathrm{GHz}$, are $\mathrm{a}_{0}=0.105 \mathrm{~m}, \mathrm{e}_{0}=0.422, \mathrm{a}_{1}=0.6556 \mathrm{~m}$, and $\mathrm{H}=1.261 \mathrm{~m}$. These dimensions correspond roughly to a 20 -dB-gain conical horn of length $1.261 \mathrm{~m}$ fed by a cylindrical waveguide of diameter $0.2 \mathrm{~m}$ and an aperture of diameter $1.102 \mathrm{~m}$.

To test the symmetry of the field pattern of the antenna, the square of the normalized field patterns in the E- and H-planes were integrated over the main lobe (ML) for different values of the eccentricity of the aperture $e_{1}$. The dependence of

$$
\mathrm{DEH}=\int_{\mathrm{ML}}(|\mathrm{E}(\theta, \phi=0)|-|\mathrm{E}(\theta, \phi=\pi / 2)|)^{2} \mathrm{~d} \theta
$$

on $e_{1}$ is shown in Fig. 2. Note that as $e_{1}$ is varied, the semi-major axis of the aperture is kept constant. It can be clearly seen that a minimum is attained for an eccentricity in the neighborhood of $e_{1}=0.67$.

Fig. 3 shows the normalized field patterns in the E- and $H$-planes when $e_{1}=0.67$. All other dimensions are kept equal to those inFig. 2 . The reasonably high degree of symmetry of the radiation pattern of the horn is evident.

\section{CONCLUSIONS}

Elliptic horn antennas fed by elliptic waveguides of varying eccentricities were analyzed taking into account the true propagation constant inside the horn. It is demonstrated that the asymmetry, which exists in the field pattern of a conical horn, is greatly reduced when an elliptic horn with optimized eccentricity is used.

\section{REFERENCES}

1. Balanis C. A., Antenna Theory: Analysis and Design, John Wiley\& Sons, New York, 1982.

2. N. W. McLachlan, Theory and Applications of Mathieu Functions, Oxford, Clarendon, 1947.

3. S.-J. Zhang and Y.-C. Shen, "Eigenmode sequence for an elliptical waveguide with arbitrary ellipticity," IEEE Trans, MTT., Vol. 43, pp. 227-230, Jan. 1995.

4. J. Bornemann, "Modified analysis of conical horns," Microwave Opt. Technol. Lett., Vol. 10, pp. 91-94, Oct. 1995. 


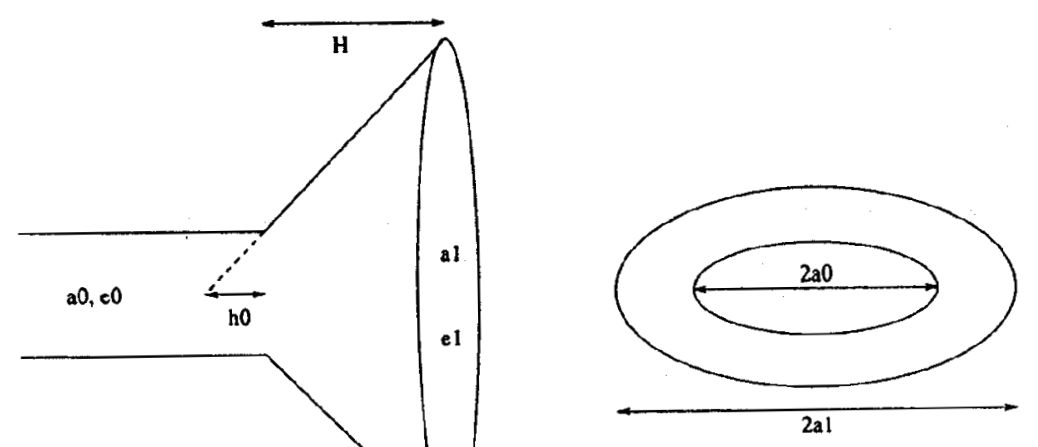

Fig.1 Geometry of elliptic horn.

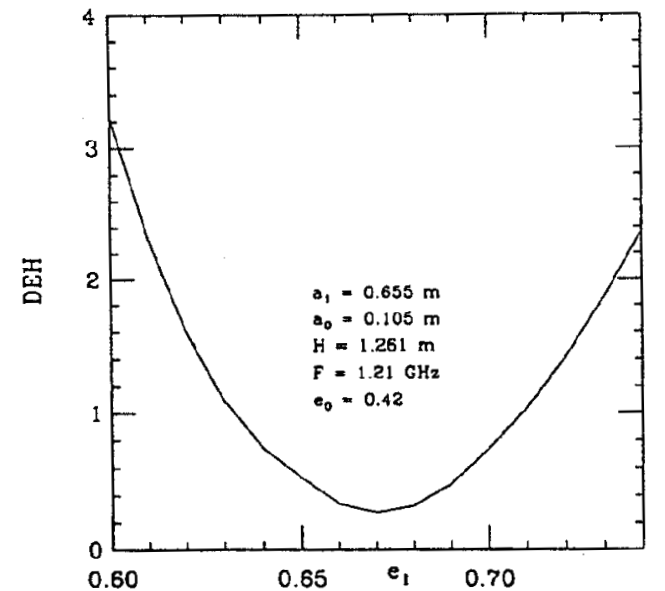

Fig.2 DEH versus aperture eccentricity $\mathrm{e}_{\mathrm{l}}$.

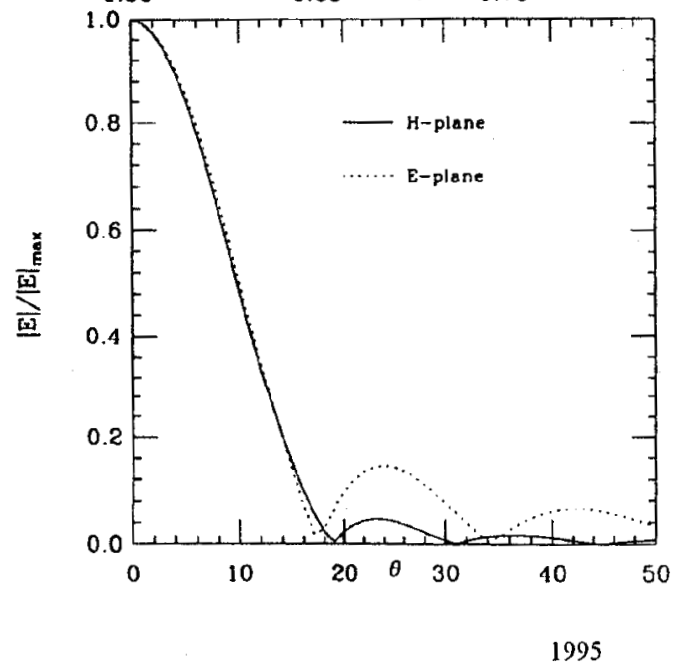

Fig.3 E- and H-plane patterns for $e_{1}=0.67$.

1995 